\title{
In-situ preparation of MOFs/SiC/PVA-Co-PE nanofiber membranes for efficient photocatalytic reduction of $\mathrm{CO}_{2}$
}

\author{
Xianwei Fan ${ }^{1}$, Ran Zhao ${ }^{1, *}$, Haoxuan $\mathrm{Hu}^{1}$, Junyi Liu ${ }^{1}$, Yahui Nie ${ }^{1}$, and Dong $\mathrm{Wang}^{1 *}$ \\ ${ }^{1}$ Hubei Key Laboratory of Advanced Textile Materials \& Application, Hubei International Scientific and Technological Cooperation Base \\ of Intelligent Textile Materials \& Application, Wuhan Textile University, Wuhan 430200, China.
}

\begin{abstract}
In this paper, we prepared a polyvinyl alcohol-polyethylene (PVA-Co-PE) composite nanofiber membrane catalyst decorated by $\mathrm{Fe}-\mathrm{MOF} / \mathrm{SiC}$ and completed the photocatalytic reduction of $\mathrm{CO}_{2}$ performance. The results show that the $\mathrm{CO}_{2}$ conversion rate of composite film materials under visible light irradiation is increased by 31 times compared with powdered $\mathrm{Fe}-\mathrm{MOF} / \mathrm{SiC}$ materials. Through SEM, XRD, BET, FTIR, DRS and other characterization methods, the influencing factors of the photocatalytic $\mathrm{CO}_{2}$ reduction process of the composite nanofiber membrane were investigated. The synergistic effect of $\mathrm{Fe}-\mathrm{MOFs} / \mathrm{SiC}$ and nanofiber membrane photocatalysis is beneficial to electron-hole pairs. The effective separation and strong absorption in the visible light region make it exhibit excellent photocatalytic activity in the photocatalytic $\mathrm{CO}_{2}$ reduction reaction.
\end{abstract}

\section{Introduction}

The rapid consumption of fossil fuels produces excessive carbon dioxide which is the main cause of climate warming ${ }^{1-4}$. To alleviate the aforementioned environmental problems, people have made a lot of efforts in the capture, storage and utilization of carbon dioxide $^{5}$. At present, the conversion of carbon dioxide into high value-added chemical fuel storage and utilization is in line with the development concept of modern and sustainable industrialization ${ }^{6}$. However, the huge input energy required for $\mathrm{C}=\mathrm{O}$ in the $\mathrm{CO}_{2}$ conversion process is now the biggest challenge ${ }^{7-9}$. In this case, as an alternative sustainable method, the use of free and abundant solar energy for photocatalytic reduction of $\mathrm{CO}_{2}$ is very attractive. Iron-based metal-organic framework materials are very promising catalysts in the field of photocatalysis, thanks to their large surface area ${ }^{10}$, adjustable structure ${ }^{11}$, high porosity ${ }^{12}$, adjustable light collection capacity in a wide range and uniformly distributed catalytic activity sites and their accessibility to catalysis ${ }^{13-14}$. However, the poor stability of the metal-organic framework itself is an important reason for limiting the photocatalytic activity.

Silicon carbide is a typical one-dimensional nanomaterial, which has high stability and lightweight, but its wide bandgap limits the photocatalytic activity ${ }^{15-19}$ The mixing of silicon carbide and MOF can obtain better activity and particularly effective active sites, which not only inherits the advantages of MOFs to a certain extent but also provides other advantages that the parent MOFs may not possess. However, the recycling problem is still a huge challenge, which will lead to reduced catalytic activity, reuse, and secondary pollution problems.
Combining photocatalysts and nanofiber materials to form hybrid membranes is a good way to recover photocatalysis. Fixing nanoparticles on a fiber carrier has several advantages: (1) It avoids expensive separation steps; (2) Gives the membrane substrate self-cleaning performance; (3) The synergistic effect of membrane separation and photocatalytic reaction ${ }^{20-25}$.

Recently, our group has developed a simple method to prepare polyvinyl alcohol-ethylene-polyethylene (PVA-Co-PE) nanofibers. Polyvinyl alcohol-ethylene copolymer is a commercial polymer with abundant active hydroxyl groups, which is convenient for activation and modification. In this paper, $\mathrm{Fe}-\mathrm{MOF} / \mathrm{SiC}$ was prepared by in-situ synthesis method and loaded on active PVA-Co-PE film, which showed excellent photocatalytic activity under visible light irradiation.

\section{Experimental section}

\subsection{Synthesis of MOFs/SiC}

Disperse $\mathrm{SiC}$ nanowires $(15 \mathrm{mg})$ in $40 \mathrm{~mL}$ ethanol and sonicate for 30 minutes. Then, $0.85 \mathrm{~g} \mathrm{FeCl}_{3} \cdot 6 \mathrm{H}_{2} \mathrm{O}$ was added to the dispersion and stirred for 1 hour. Afterward, a solution of 2-methylimidazole $(2.78 \mathrm{~g})$ in anhydrous methanol $(10 \mathrm{ml})$ was added dropwise to the above mixture and stirred at room temperature for $5 \mathrm{~h}$, washed with ethanol four times, and vacuum dried at $40^{\circ} \mathrm{C}$ for 12 $\mathrm{h}$. The dried sample was heated from room temperature to $400^{\circ} \mathrm{C}$ at a rate of $2^{\circ} \mathrm{C}$ per minute and kept in an air atmosphere for $1 \mathrm{~h}$ to obtain MOFs/SiC ${ }^{25}$. 


\subsection{Synthesis of PVA-Co-PE nanofiber suspension (PCP) and composite nanofiber membrane (MOFs/SiC/PCP)}

PVA-Co-PE nanofibers were prepared according to the method in the literature. Then weigh $15 \mathrm{~g}$ of the nanofiber membrane and dissolve it in $500 \mathrm{~mL}$ of isopropanol and deionized aqueous solution with a volume ratio of 1:1, and use a high-speed shear mixer to disperse the nanofibers in the aqueous solution to form a stable suspension ${ }^{20}$.

Take $20 \mathrm{ml}$ of the prepared PVA-Co-PE nanofiber suspension, add $20 \mathrm{wt} \% \mathrm{Fe}-\mathrm{MOFs} / \mathrm{SiC}$ powder and disperse it in the well-dissolved solution to reach equilibrium, stir for 30 minutes, and perform ultrasonic treatment for 30 minutes. The membrane obtained by suction filtration of the solution was dried in an oven at $40^{\circ} \mathrm{C}$ overnight to obtain $\mathrm{MOFs} / \mathrm{SiC} / \mathrm{PVA}-\mathrm{Co}-\mathrm{PE}$.

\section{Results and discussion}

\subsection{Photocatalytic performance experiment}

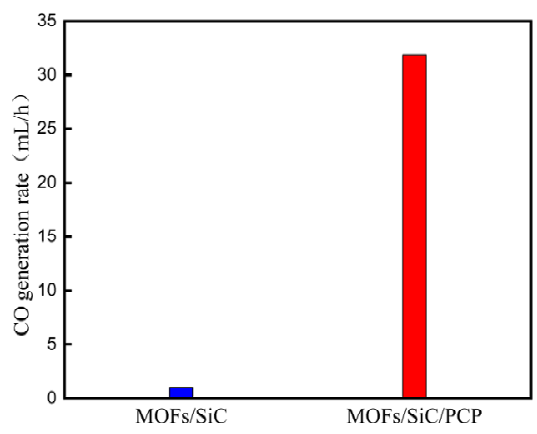

Fig. 1 Photocatalytic $\mathrm{CO}_{2}$ performance experiment Figure 1 shows the $\mathrm{CO}_{2}$ photoreduction rate of nanoparticles and nanofiber membranes under visible light irradiation. The reason for the photocatalytic activity of pure nanofiber membranes is that the specific surface area is small and the charge recombination rate is fast; while the specific surface area of $\mathrm{Fe}-\mathrm{MOF} / \mathrm{SiC}$ is large, but the bandgap itself is too high, so it is difficult for electrons to be excited from the valence band to the conduction band. Through the interface interaction between the two composite membranes, the specific surface area is increased, the bandgap is significantly reduced, and the photocatalytic activity is significantly improved.

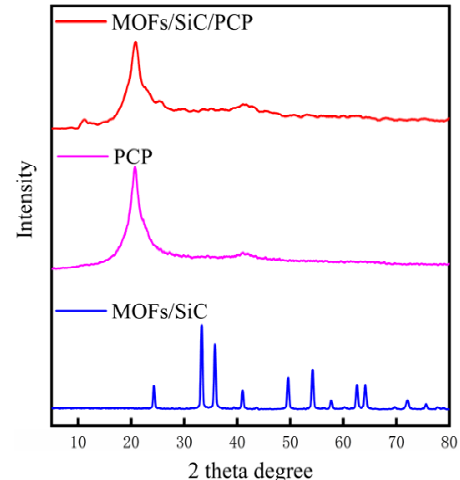

Fig. 2 XRD patterns of as-obtained catalysts

Figure 2 shows the XRD patterns of $\mathrm{MOFs} / \mathrm{SiC}, \mathrm{PCP}$ and $\mathrm{MOF} / \mathrm{SiC} / \mathrm{PCP}$. The results show that in the process of preparing $\mathrm{MOF} / \mathrm{SiC} / \mathrm{PCP}, \mathrm{MOF} / \mathrm{SiC} / \mathrm{PCP}$ retains two characteristic peaks of $\mathrm{MOF} / \mathrm{SiC}$ and $\mathrm{PCP}$, and the intensity of characteristic peaks is due to the interface interaction between MOFs/SiC and PCP. It is reduced to a certain extent, which indicates the successful preparation of $\mathrm{MOF} / \mathrm{SiC}$ composite nanofiber film photocatalyst.

\subsection{SEM}

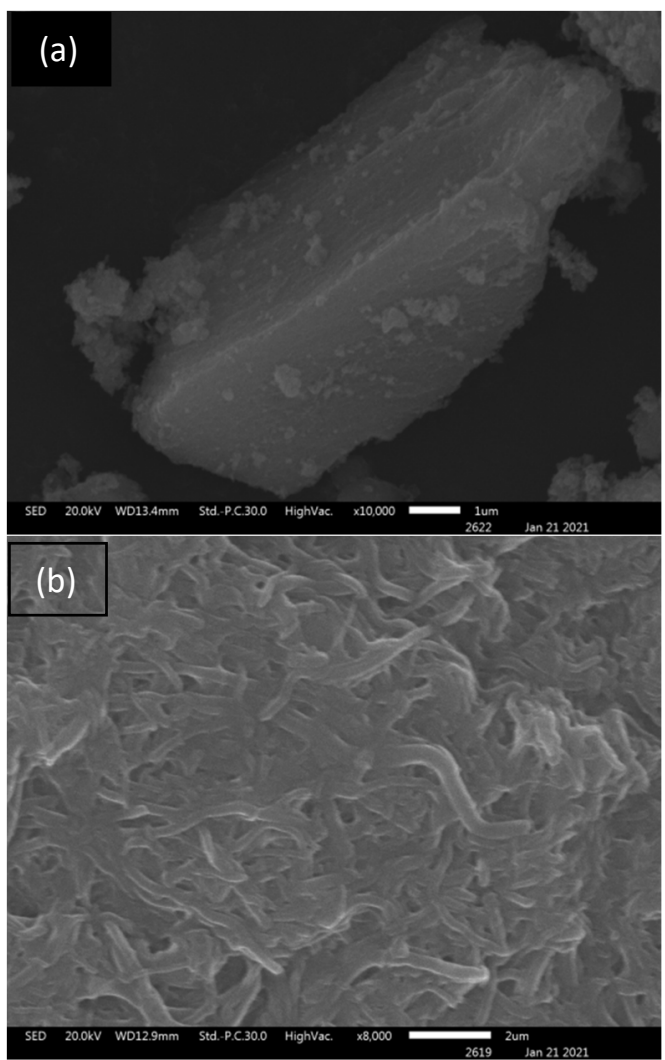

\subsection{XRD}




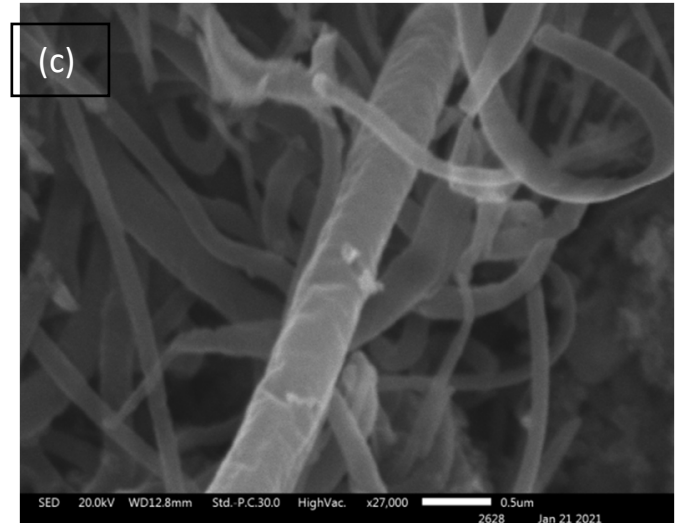

Fig. 3 SEM patterns of (a) MOFs/SiC; (b) PCP and (c) $\mathrm{MOF} / \mathrm{SiC} / \mathrm{PCP}$

Figure 3 is the SEM spectra of MOFs/SiC, PCP and $\mathrm{MOF} / \mathrm{SiC} / \mathrm{PCP}$. MOFs/SiC nanoparticles are anchored on the surface of the nanofiber membrane through a fixed layer, and the fiber morphology of the composite nanofiber membrane can be seen, and the fibers are randomly distributed with a porous structure inside. After the MOFs/SiC is fixed to the fiber matrix, the wrinkles appear on the surface of the fiber, and MOFs/SiC are uniformly distributed throughout the film matrix.

\subsection{FTIR}

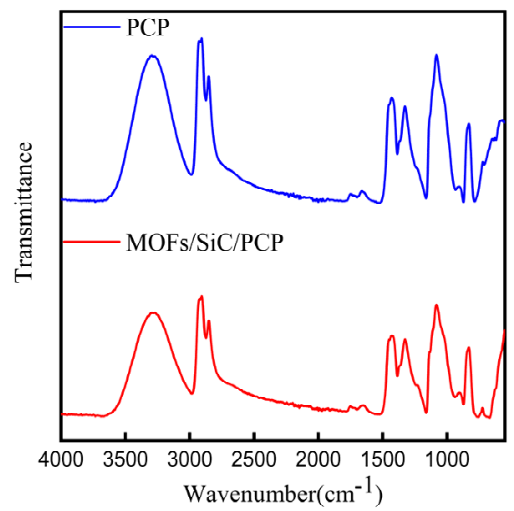

Fig. 4 FTIR curves of MOFs/SiC, MOFs/SiC/PCP

According to the FTIR spectroscopy results in Figure 4, the characteristic peaks at $571 \mathrm{~cm}^{-1}$ correspond to the tensile vibrations of $\mathrm{Fe}-\mathrm{O}$, respectively. The enhanced $\mathrm{CO}_{2}$ reduction effect is mainly since $\mathrm{Fe}-\mathrm{O}$ clusters can directly absorb visible light and transfer electrons. The direct excitation of $\mathrm{Fe}-\mathrm{O}$ clusters in the iron-based metal-organic framework causes electrons to transfer from $\mathrm{O}^{2-}$ to $\mathrm{Fe}^{3+}$ to form $\mathrm{Fe}^{2+}$, which is the key to photocatalytic $\mathrm{CO}_{2}$ reduction. The peaks of 3200 $\mathrm{cm}^{-1} \sim 3500 \mathrm{~cm}^{-1}$ and $2800 \mathrm{~cm}^{-1} \sim 2900 \mathrm{~cm}^{-1}$ correspond to the hydroxyl association peak and the methylene tensile vibration peak, respectively, indicating that $\mathrm{MOFs} / \mathrm{SiC}$ has been successfully loaded on the surface of the nanofiber membrane.
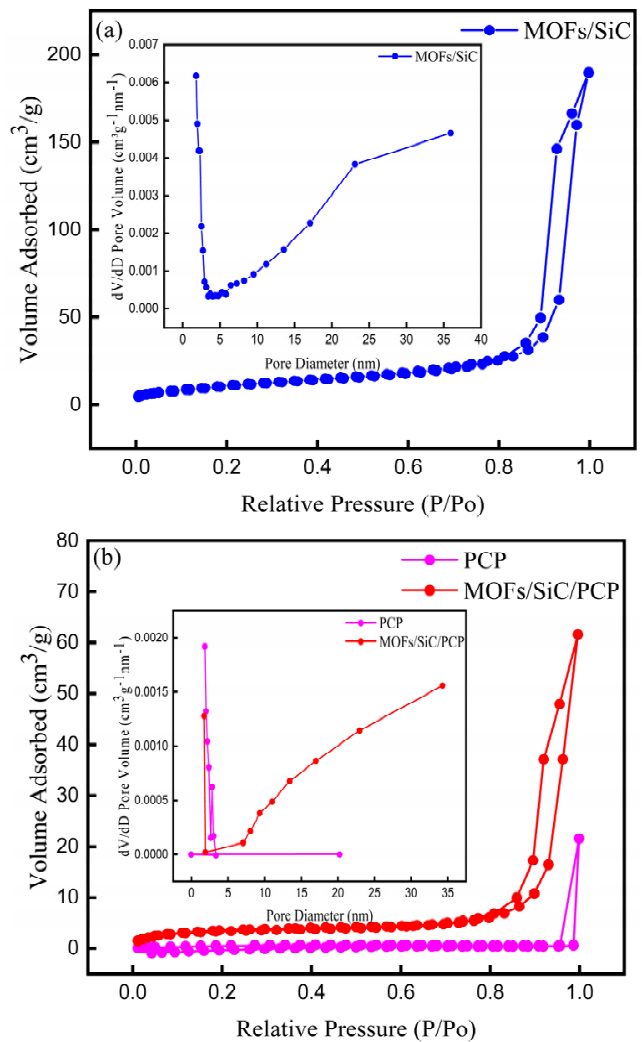

Fig. $5 \mathrm{~N}_{2}$ adsorption-desorption isotherm of (a) MOFs/SiC; (b) $\mathrm{PCP}$ and MOFs/SiC/PCP and corresponding BJH PSD curves (inset).

Table1. Textural Properties of MOFs/SiC, PCP, and $\mathrm{MOFs} / \mathrm{SiC} / \mathrm{PCP}$

\begin{tabular}{|c|c|c|c|}
\hline sample & $\mathrm{S}_{\text {BET }}\left(\mathrm{m}^{2} \mathrm{~g}^{-1}\right)$ & $\mathrm{V}_{\text {total }}\left(\mathrm{cm}^{3} \mathrm{~g}^{-1}\right)$ & $\mathrm{D}(\mathrm{nm})$ \\
\hline $\mathrm{MOFs} / \mathrm{SiC}$ & 40.4 & 0.2886 & 28.592 \\
\hline $\mathrm{PCP}$ & 2.3 & 0.0234 & 40.110 \\
\hline $\begin{array}{c}\mathrm{MOFs} / \mathrm{SiC} / \mathrm{PC} \\
\mathrm{P}\end{array}$ & 11.3 & 0.0937 & 33.084 \\
\hline
\end{tabular}

Figure 5 shows the $\mathrm{N}_{2}$ adsorption-desorption isotherms of $\mathrm{MOFs} / \mathrm{SiC}$, nanofiber membranes and composite nanofiber membranes. Combining the pore size distribution diagram in the illustration and the parameter analysis in the table, it is found that the pore size of the nanofiber composite membrane has changed significantly compared with the pure nanofiber membrane, exposing more surface active sites, thereby enhancing the photocatalytic ability. Due to multiple reflections, the porous structure can also promote light absorption by enhancing visible light scattering in the cavity. This indicates that the increase in adsorption capacity helps to improve the conversion efficiency of photocatalytic $\mathrm{CO}_{2}$ reduction.

\subsection{DRS}

\subsection{BET}



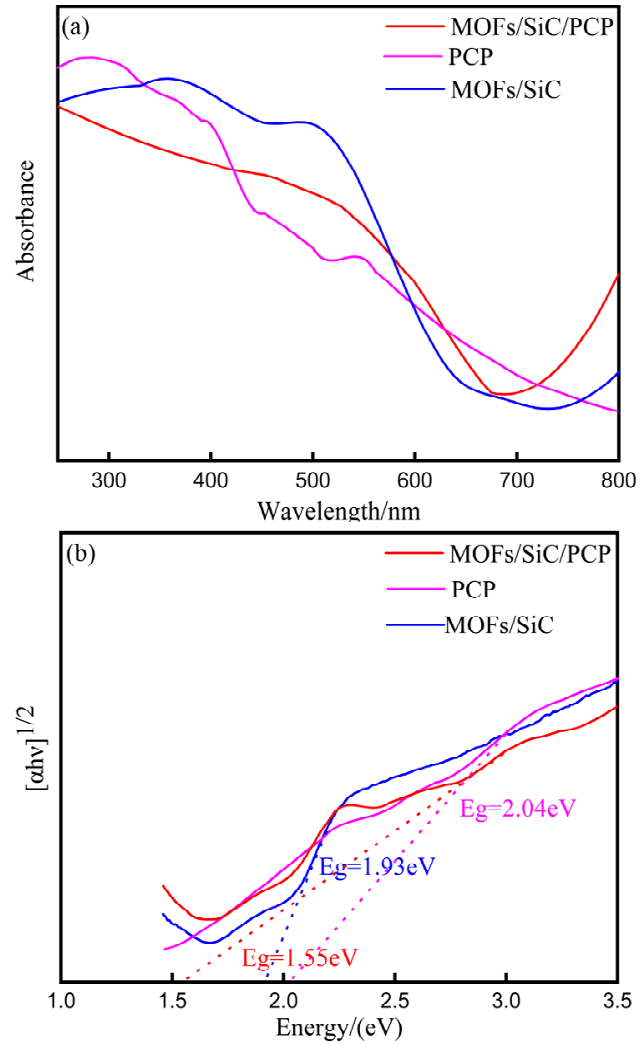

Fig. 6 (a) DRS spectra of MOFs/SiC, PCP, MOFs/SiC/PCP and; (b) plots of $(\alpha h v)^{1 / 2}$ as a function of vs. photon energy of $\mathrm{MOF} / \mathrm{SiC}, \mathrm{PCP}, \mathrm{MOF} / \mathrm{SiC} / \mathrm{PCP}$.

Figure 6 shows that MOFs/SiC, PCP and $\mathrm{MOF} / \mathrm{SiC} / \mathrm{PCP}$ all have high visible light absorption. Compared with the nanoparticle sample, the light absorption of the composite nanofiber membrane is significantly increased. Besides, a redshift of the absorption edge was observed in the composite nanofiber membrane. These data further indicate that $\mathrm{Fe}, \mathrm{C}$ and $\mathrm{O}$ have been successfully doped into the crystal structure of the nanofiber membrane, which is consistent with the XRD data. The bandgap of the composite nanofiber membrane calculated by the Kubelka-Munk method is $1.55 \mathrm{eV}$, which is lower than the bandgap of the nanoparticle sample $(1.93 \mathrm{eV})$ and the bandgap of the pure nanofiber membrane $(2.04 \mathrm{eV})$. The interfacial interaction between composite nanofiber membranes provides an effective channel for rapid charge transfer during photochemical reactions.

\subsection{PL}

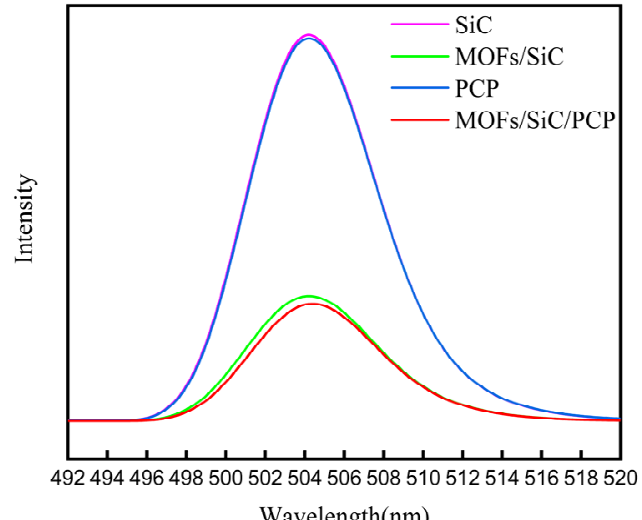

Fig. 7 Photoluminescence spectra of $\mathrm{MOFs} / \mathrm{SiC}, \mathrm{PCP}$, $\mathrm{MOFs} / \mathrm{SiC} / \mathrm{PCP}$.

As shown in Figure 7, the PL spectrum shows similar peaks with different intensities for the three materials. The emission peak at $503 \mathrm{~nm}$ was observed by $500 \mathrm{~nm}$ light excitation, which proved the recombination of light-excited electron-hole pairs. Compared with the nanofiber membrane sample, the emission peak intensity of the composite nanofiber membrane is greatly reduced, indicating that the interface interaction between the two is beneficial to improve the light separation efficiency.

\section{Conclusion}

1) The iron-based metal-organic framework composite nanofiber membrane photocatalyst was prepared by in-situ polymerization. The MOFs/SiC/PCP composite film shows excellent photocatalytic performance. The $\mathrm{CO}$ generation rate reaches $31.9 \mathrm{~mL} / \mathrm{h}$, which is 31 times higher than that of MOFs/SiC nanoparticles. It also solves the problem of photocatalyst difficult to recycle and secondary pollution.

2) According to the results of XRD, SEM, BET, DRS, PL, FTIR, after doping with MOFs/SiC, the light absorption range of the pure nanofiber film becomes wider and the bandgap is significantly reduced, which indicates that the doping of nanoparticles is beneficial to the separation of carriers.

3) The iron-based metal-organic framework film has high photocatalytic activity, which can be attributed to the unique structure with unsaturated coordination metal sites, excellent selectivity, and effective electron transfer. Therefore, the use of iron-based metal-organic framework films as photocatalytic reduction gas-phase reactions has great application prospects.

\section{References}

1. Humphrey, V. Zscheischler, J. Ciais, P. Gudmundsson, L. Sitch, S. Seneviratne, S. I.,Nature, 560, 628-631,(2018).

2. Soden, B. J. Collins, W. D. Feldman, D. R.,Science, 361, 326-327,(2018).

3. Ding, M. Jiang, H.-L.,ACS Catalysis, 8, 3194-3201,(2018). 
4. Hou, S.-L. Dong, J. Jiang, X.-L. Jiao, Z.-H. Zhao, B.,Angew. Chem. Int. Ed., 58, 577-581,(2019).

5. An, B. Li, Z. Song, Y. Zhang, J. Zeng, L. Wang, C. Lin, W.,Nature Catalysis, 2, 709-717,(2019).

6. Li, F., et al.,Nature Catalysis, 3, 75-82,(2020).

7. Li, X. Yu, J. Jaroniec, M. Chen, X.,Chem. Rev., 119, 3962-4179,(2019).

8. Ding, M. Cai, X. Jiang, H.-L.,Chemical Science, 10, 10209-10230,(2019).

9. Jiao, L. Seow, J. Y. R. Skinner, W. S. Wang, Z. U. Jiang, H.-L.,Mater. Today, 27, 43-68,(2019).

10. Dong, H. Zhang, X. Lu, Y. Yang, Y. Zhang, Y.-P. Tang, H.-L. Zhang, F.-M. Yang, Z.-D. Sun, X. Feng, Y.,Applied Catalysis B: Environmental, 276, 119173,(2020).

11. Dao, X.-Y. Guo, J.-H. Wei, Y.-P. Guo, F.; Liu, Y. Sun, W.-Y.,Inorg. Chem., 58, 8517-8524,(2019).

12. Dao, X.-Y. Xie, X.-F. Guo, J.-H. Zhang, X.-Y. Kang, Y.-S. Sun, W.-Y.,ACS Applied Energy Materials, 3, 3946-3954,(2020).

13. Reddy, C. V. Reddy, K. R. Harish, V. V. N. Shim, J. Shankar, M. V. Shetti, N. P. Aminabhavi, T. M.,Int. J. Hydrogen Energy, 45, 7656-7679,(2020).

14. Liu, N. Tang, M. Wu, J. Tang, L. Huang, W. Li, Q. Lei, J. Zhang, X. Wang, L.,Advanced Materials Interfaces, 7, 2000468,(2020).
15. Wang, Y. Zhang, L. Zhang, X. Zhang, Z. Tong, Y. Li, F. Wu, J. C. S. Wang, X.,Applied Catalysis B: Environmental, 206, 158-167,(2017).

16. Weng, W. Wang, S. Xiao, W. Lou, X. W.,Adv. Mater., 32, 2001560,(2020).

17. Han, C. Wang, B. Wu, C. Shen, S. Zhang, X. Sun, L. Tian, Q. Lei, Y. Wang, Y.,ChemistrySelect, 4, 2211-2217,(2019).

18. Wang, Y., et al.,J. Am. Chem. Soc., 140, 14595-14598,(2018).

19. Han, C. Lei, Y. Wang, B. Wang, Y.,ChemSusChem, 11, 4237-4245,(2018).

20. Chen, J. Pakdel, E. Xie, W. Sun, L. Xu, M. Liu, Q. Wang, D.,Advanced Fiber Materials, 2, 64-73,(2020).

21. Ni, Y. Yan, K. Xu, F. Zhong, W. Zhao, Q. Liu, K. Yan, K. Wang, D.,Composites Communications, 12, 112-116,(2019).

22. Sun, H. Zhou, Q. Zhao, Q. Shi, L. Wang, D. Mei, T.,Composites Communications, 13, 151-155,(2019).

23. Cheng, P. Liu, Y. Yi, Z. Wang, X. Li, M. Liu, Q. Liu, K. Wang, D.,Composites Communications, 9, 11-16,(2018).

24. Zhu, Q. Li, Y. Wang, W. Sun, G. Yan, K. Wang, D.,Composites Communications, 10, 36-40,(2018).

25. Zhu, Q. Wang, Y. Li, M. Liu, K. Hu, C. Yan, K. Sun, G. Wang, D.,Sep. Purif. Technol., 186, 70-77,(2017). 